\title{
Técnicas de refuerzo en asignaturas universitarias que emplean conceptos matemáticos
}

\section{Carlos Mascaraque Ramírez ${ }^{a}$, Lorena Para González ${ }^{\mathrm{b}}$ y Juan Eugenio Para Conesa ${ }^{\mathrm{c}}$}

appto. Física Aplicada y Tecnología Naval, Universidad Politécnica de Cartagena, carlos.mascaraque@upct.es, bDpto. Ciencias Jurídicas y Económicas, Universidad Isabel I, lorena.para@uil.es, Dpto. Ingeniería Mecánica, Materiales y Fabricación, Universidad Politécnica de Cartagena,je.para@upct.es.

\begin{abstract}
Students of technical degrees identify the basic need to apply mathematical concepts in multiple subjects, using mathematical tools to solve problems in other areas. At this point it has been verified that there is a difficulty on the part of the students to recognize which mathematical knowledge they must use in each proposed exercise and problem. Therefore, the assimilation of the competences proper to each subject is worsened.
\end{abstract}

This research makes a series of proposals to mitigate this problem and offer teachers techniques that encourage student learning. By means of surveys completed by the students, the different techniques proposed will be evaluated, thus proposing a range of solutions within the reach of the teaching staff, capable of adapting them to the different subjects of the degrees, to the profile of the students, and to the needs of the teacher.

Keywords: assimilation of mathematical concepts, competences, university teaching, engineering degrees.

\section{Resumen}

El alumnado de las titulaciones técnicas identifica la necesidad básica de aplicar conceptos matemáticos en múltiples asignaturas, utilizando las herramientas matemáticas para resolver los problemas del resto de áreas. En este punto se ha comprobado que existe una dificultad por parte de los estudiantes de reconocer qué conocimientos matemáticos han de utilizar en cada ejercicio y problema propuesto. Como consecuencia, se ve empeorada la asimilación de las competencias propia de cada asignatura.

La presente investigación realiza una serie de propuestas para mitigar esta problemática y ofrecer al docente técnicas que fomenten el aprendizaje de los estudiantes. Por medio de encuestas completadas por los estudiantes, se valorarán las diferentes técnicas planteadas, proponiendo así un abanico de soluciones al alcance del profesorado, capaz de adaptarlas a las asignaturas de los títulos, al perfil de los estudiantes, y a las necesidades del docente.

Palabras clave: asimilación de conceptos matemáticos, competencias, docencia universitaria, estudios de ingeniería. 


\section{Introducción}

En los estudios técnicos universitarios, como son los estudios de Grado en Arquitectura Naval e Ingeniería de Sistemas Marinos y Máster en Ingeniería Naval y Oceánica, es usual que múltiples asignaturas tengan una fuerte base matemática, donde para resolver cuestiones y problemas de áreas como la construcción naval, organización de empresas, ingeniería de fabricación, mecánica, etc. sea necesario aplicar conocimientos del área de las matemáticas. Es por ello, que los alumnos tienen en sus programaciones diferentes asignaturas de base matemática, impartidas por profesores de las áreas de matemática aplicada o estadística, como se puede ver en los planes de estudio y memorias de estas titulaciones (ETSINO, 2019)

Cuando los alumnos están cursando los últimos años del grado o los estudios de máster, han superado múltiples asignaturas de matemáticas, y se puede decir que están en un momento álgido de su conocimiento matemático (Luaces, 1998), donde diferentes autores han investigado sobre las mejores metodologías para enseñarles los conceptos matemáticos a los estudiantes de ingeniería (Pohjolainen et al., 2018), analizando las ventajas que el pensamiento matemático les ofrece (Serna y Flórez, 2013) o el papel que la modelización matemática les aporta durante sus estudios (Brito-Vallina et al., 2011), así como en la didáctica de esta ciencia, es decir, cómo enseñarle matemáticas a un futuro ingeniero (Harris et al., 2015). La siguiente cuestión, que es la que aborda esta investigación, es cómo ayudar al estudiante a que aplique sus conocimientos matemáticos para resolver problemas de ingeniería (Godino et al., 2014).

En este aspecto, algunas investigaciones actuales han identifica una problemática actual en que bastante interesante para profundizar en ella, y es el siguiente problema: los alumnos tienen dificultades de aplicar sus conocimientos matemáticos a la hora de resolver problemas de asignaturas no afines al área de las matemáticas. Es decir, saben emplear las herramientas matemáticas en las asignaturas propias de éste área, pero no saben exportar estas herramientas a otros contextos. Sobre esta temática se ha profundizado en algunas investigaciones previas, definiendo una serie de técnicas de mejora para la asimilación de los conceptos matemáticos (Mascaraque-Ramírez y Para-González, 2016), donde también se ha visto lo importante que es el contexto de aplicación, con el objetivo de que el alumno no pierda la perspectiva del uso de sus conocimientos matemáticos (Mendible y Ortiz, 2007). Todo este proceso debe estar enfocado a los dos actores principales, al profesorado y al alumnado, aportándole herramientas al profesorado para facilitar su labor (Barrera, Pulido y González, 2018, Avilés, Rodríguez y Solano, 2018) y estudiando la respuesta del alumnado, analizando si mejora su aprendizaje y conociendo su opinión con la finalidad de una mejora continua de las clases impartidas (Napitupulu et al., 2018) y se optimice la adquisición de las competencias propias de la titulación cursada (Manzueta Concepción, Machado Ramírez y Blanco Sánchez, 2018)

Con todo ello, es necesario analizar las posibles soluciones para apaliar este efecto, ofreciendo un amplio abanico de técnicas a los docentes, valorándolas para conocer cuáles son las más idóneas para los alumnos, con la finalidad última de que los estudiantes consoliden las competencias que el titulo comprende (Arraiz et al., 2016). 


\section{Objetivos}

Esta investigación se enfoca a la necesidad de que el alumnado identifique correctamente las herramientas matemáticas que ha de aplicar en los problemas planteados en asignaturas que no son del área de las matemáticas, permitiéndole así asimilar correctamente las competencias de dichas asignaturas.

Para conseguir cubrir esta necesidad, este proyecto de innovación docente persigue identificar una sería de técnicas que permitan a los estudiantes repasar los conceptos matemáticos al comienzo de las asignaturas de la forma más adecuada posible, y obtener una valoración por parte de los alumnos de las técnicas propuestas, para así poder elegir por parte del profesorado la que mejor se adapte a su asignatura y a las necesidades de los estudiantes.

\section{Desarrollo de la innovación}

Una vez definidos los objetivos que persigue la presente investigación, la cual está enmarcada dentro de una serie de proyectos de innovación docente que llevan realizándose desde el año 2015, se definen una serie de fases para poder cubrir todos los aspectos requeridos para el correcto estudio de la problemática planteada, estas fases son:

- Identificar técnicas que permitan repasar los conceptos matemáticos al comienzo de las asignaturas de la forma más adecuada posible

- Desarrollo de unas encuestas para poder recopilar información del alumnado.

- Obtención de resultados, con una base de datos suficientemente amplia, que permita su valoración.

- Exposición de los resultados y de conclusiones.

El seguimiento de estas fases permitirá conocer la perspectiva y la valoración de los estudiantes, pudiendo cuantificarlas por medio de diferentes técnicas de análisis de los datos registrados, lo que permitirá poder analizar los datos y valorar las diferentes técnicas planteadas para los docentes.

\subsection{Identificar técnicas que permitan repasar los conceptos matemáticos}

Por medio de la creación de un proyecto de innovación docente soportado por la Universidad Politécnica de Cartagena, un equipo de profesores ha trabajado de forma directa desarrollando cuatro posibles soluciones para mejorar la consolidación de las competencias matemáticas en los alumnos de ingeniería. El grupo docente ha trabajado junto con otros profesores que imparten docencia en la titulación, y por medio de conversaciones no estructuradas han planteado las siguientes 4 posibilidades de solución:

- Formularios matemáticos, donde el profesor facilita unos formularios matemáticos básicos para la resolución de los problemas de la asignatura. 
- Introducción matemática, donde el docente emplea tiempo de las clases para realizar una introducción matemática a los diferentes temas que se aborden a lo largo del curso en esta materia.

- Ejercicios matemáticos propuestos y resueltos, donde el docente entrega una relación de ejercicios resueltos a los alumnos, previamente al temario de la asignatura donde se precise el uso de alguna herramienta matemática, en este caso el alumno debe trabajar en casa para repasar los conceptos.

- Material audiovisual, donde el profesor debe realizar material en forma de video didácticos explicando las herramientas matemáticas requeridas en la asignatura, $\mathrm{y}$ el alumno visualizarlos previamente al temario de la asignatura.

Hay que comprender, que el profesor que va a desarrollar este tipo de contenido no es un profesor del área de matemáticas, ni afines a esta, siendo de otras áreas de las que imparten docencia en la titulación, por lo que supone un esfuerzo añadido al trabajo del propio profesor.

\subsection{Definición y desarrollo de las encuestas a los alumnos}

Con la finalidad de conocer la opinión del alumnado sobre las cuatro opciones planteadas, se ha decidido desarrollar una encuesta formada por una serie de preguntas sencillas, las cuales serán valoradas por los alumnos en una escala Likert (Echauri, Minami y Sandoval, 2014), de 1 a 5, donde el 1 representa estar muy en desacuerdo y 5 muy de acuerdo, el 3 se deja como estado neutro. Al mismo tiempo se realiza una pregunta sobre el uso de alguna de estas 4 opciones en las asignaturas, donde los alumnos valoran si hay docentes que usen algunas de estas técnicas, en este caso se adapta la escala Likert, con la siguiente correspondencia:

- 1 : En ninguna asignatura (0)

- 2: En pocas asignaturas (1-3)

- 3: En bastantes asignaturas (4-8)

- 4: En muchas asignaturas (8-15)

- 5: Prácticamente en todas las asignaturas $(+15)$

Las encuestas fueron realizadas por alumno de tercer y cuarto curso del Grado en Arquitectura Naval e Ingeniería de Sistemas Marinos, así como alumnos de primer y segundo curso del Máster Universitario en Ingeniería Naval y Oceánica, por lo tanto, se considera que son alumnos con una madurez y veteranía en los estudios suficiente para poder valorar correctamente las preguntas expuestas en la Tabla 1. Las encuestas se han realizado durante 3 años, recopilando cerca de 100 respuestas, además de comentarios añadidos por parte del alumnado que sirve para mejorar el estudio.

Las preguntas se repiten para las cuatro opciones planteadas, siendo las que se exponen en la Tabla 1: 
Tabla 1. Preguntas de las encuestas realizadas al alumnado

\section{ID Pregunta}

\section{Los formularios matemáticos...}

1 Se entregan en asignaturas del grado para la elaboración de problemas y ejercicios

En clase y durante el tiempo de estudio le ayudan (o ayudarían) a recordar conceptos de esta materia en otras asignaturas para las cuales se necesitan dichos conceptos

Influyen (o influirían) positivamente en los resultados finales que obtiene en una asignatura no afin al área de las matemáticas

\section{Las introducciones matemáticas en clase...}

Se realizan en asignaturas del Grado previamente a la realización de problemas/ejercicios, con el fin de recordar conceptos ya explicados en asignaturas del área de Matemáticas Le ayudan (o ayudarían) a recordar conceptos de esta materia en otras asignaturas para las cuales se necesiten dichos conceptos Influyen (o influirían) positivamente en los resultados finales que el alumno obtiene en una asignatura no afín al área de las matemáticas

\section{Las relaciones de ejercicios matemáticos resueltos...}

Son entregadas en asignaturas del Grado con el fin de recordar conceptos ya explicados en asignaturas del área de Matemáticas

Le ayudan (o ayudarían) a recordar aspectos de esta materia en otras asignaturas para las cuales se necesiten estos conceptos

$$
\text { Influyen (o influirían) positivamente en los resultados finales que obtiene en una asignatura no }
$$
afin al área de las matemáticas

Los materiales audiovisuales de apoyo a las herramientas matemáticas...

Le son ofrecidos en asignaturas del Grado con el fin de recordar conceptos ya explicados en asignaturas del área de Matemáticas

Le ayudan (o ayudarían) a recordar conceptos de esta materia en otras asignaturas para las cuales se necesiten dichos conceptos

Influyen (o influirían) positivamente en los resultados finales que obtiene en una asignatura no afin al área de las matemáticas

\section{Resultados}

Con los resultados de las más de cien encuestas completadas por el alumnado, se puede proceder a la presentación y análisis de los datos. Para ello, se representa una serie de gráficas donde como dato principal se muestra la media de las respuestas obtenidas en la escala Likert explicada en el apartado 3.2, también se representa el rango de datos obtenidos, desde el mínimo al máximo, y los valores de moda y mediana de cada cuestión. 
Con toda esta información se puede realizar un estudio preliminar de la valoración que realiza el alumnado de cada una de las cuatro técnicas presentadas. Donde se puede apreciar en las figuras 1 a 4, que la media de las respuestas sobre la entrega o realización de estas técnicas está cercana al valor 2, es decir, se corresponden con la respuesta de "en pocas asignaturas (1-3)", lo que hace tener una visión de que etas técnicas son muy poco empleadas en la actualidad, valor verificado con el resto de los parámetros estadísticos, como la moda y la mediana, siendo las introducciones matemáticas las que más son empleadas por los profesores.

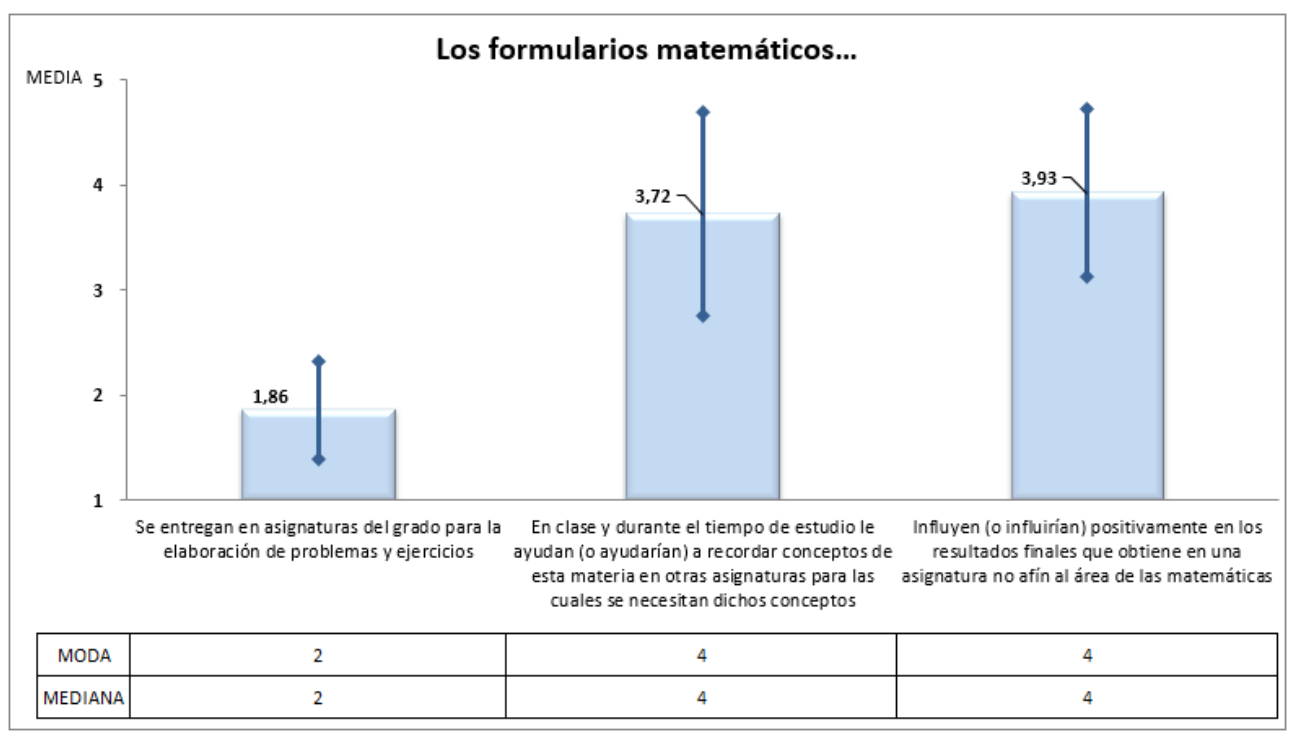

Fig. 1 Resultados de los formularios matemáticos

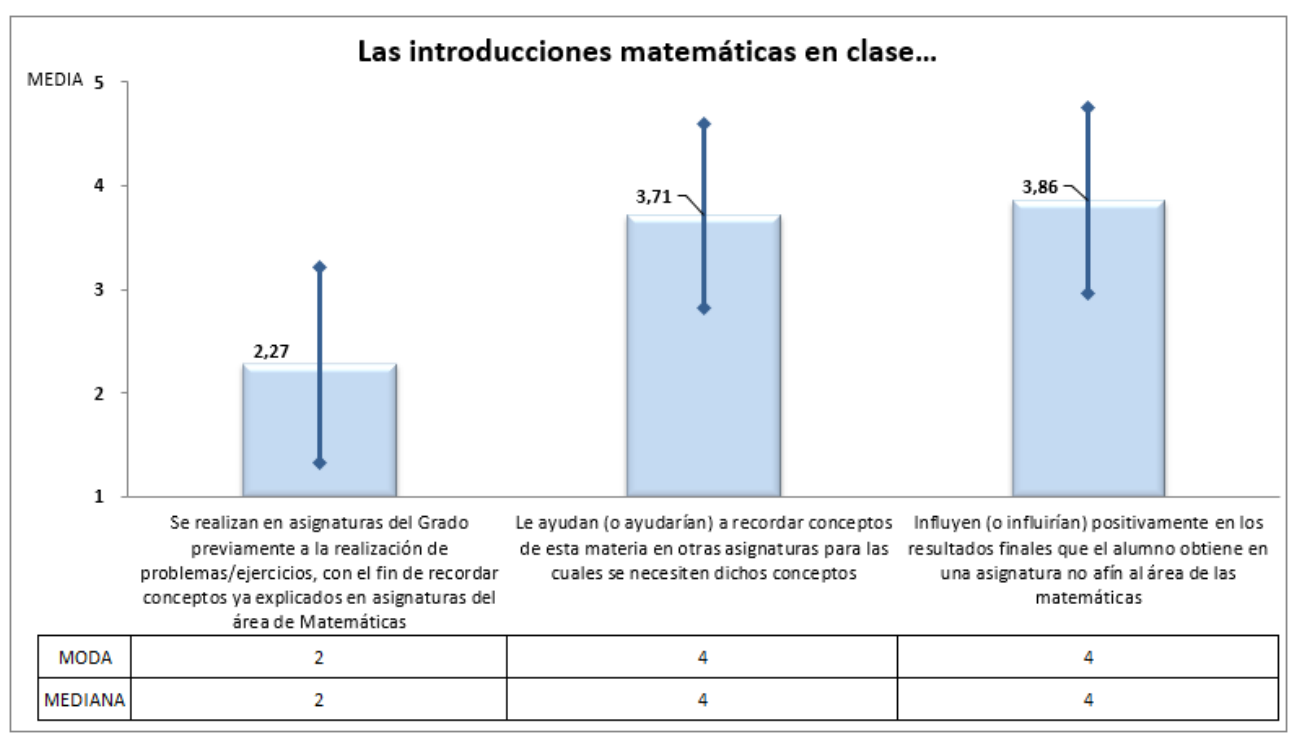

Fig. 2 Resultados de las introducciones matemáticas en clase 


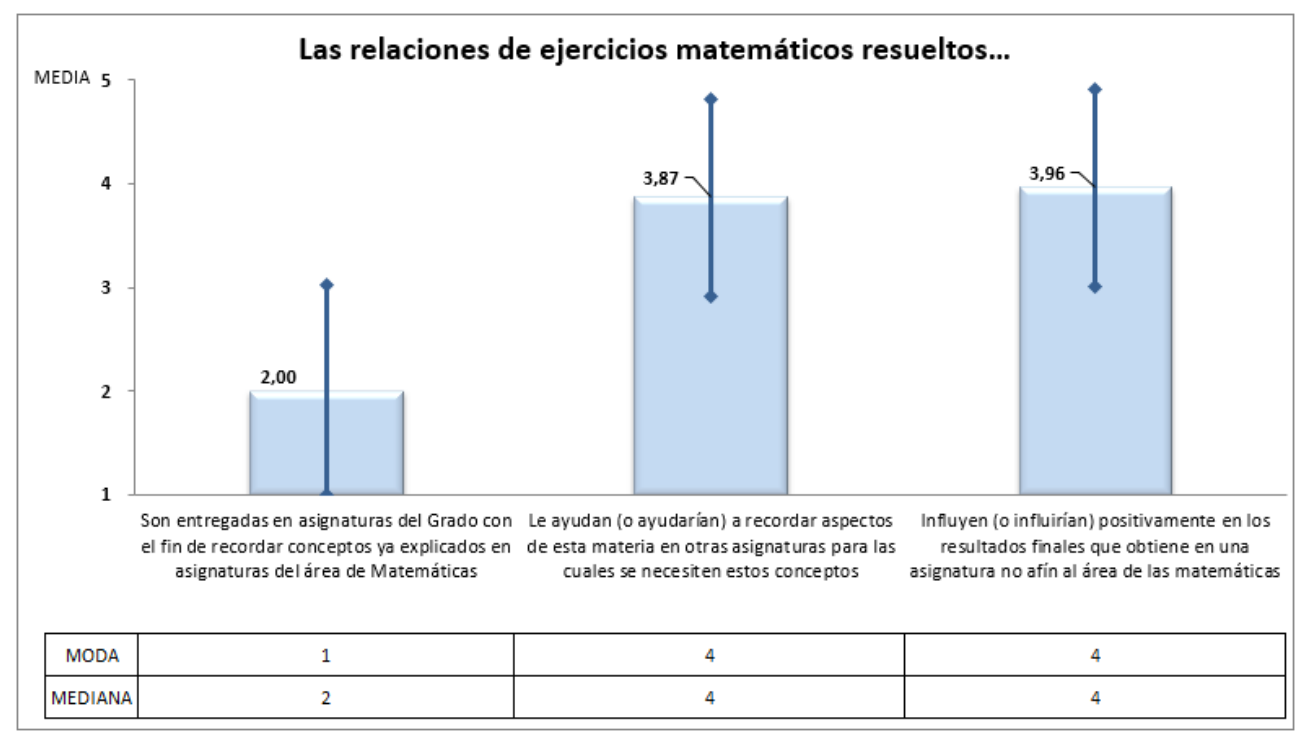

Fig. 3 Resultados de las relaciones de ejercicios matemáticos resueltos

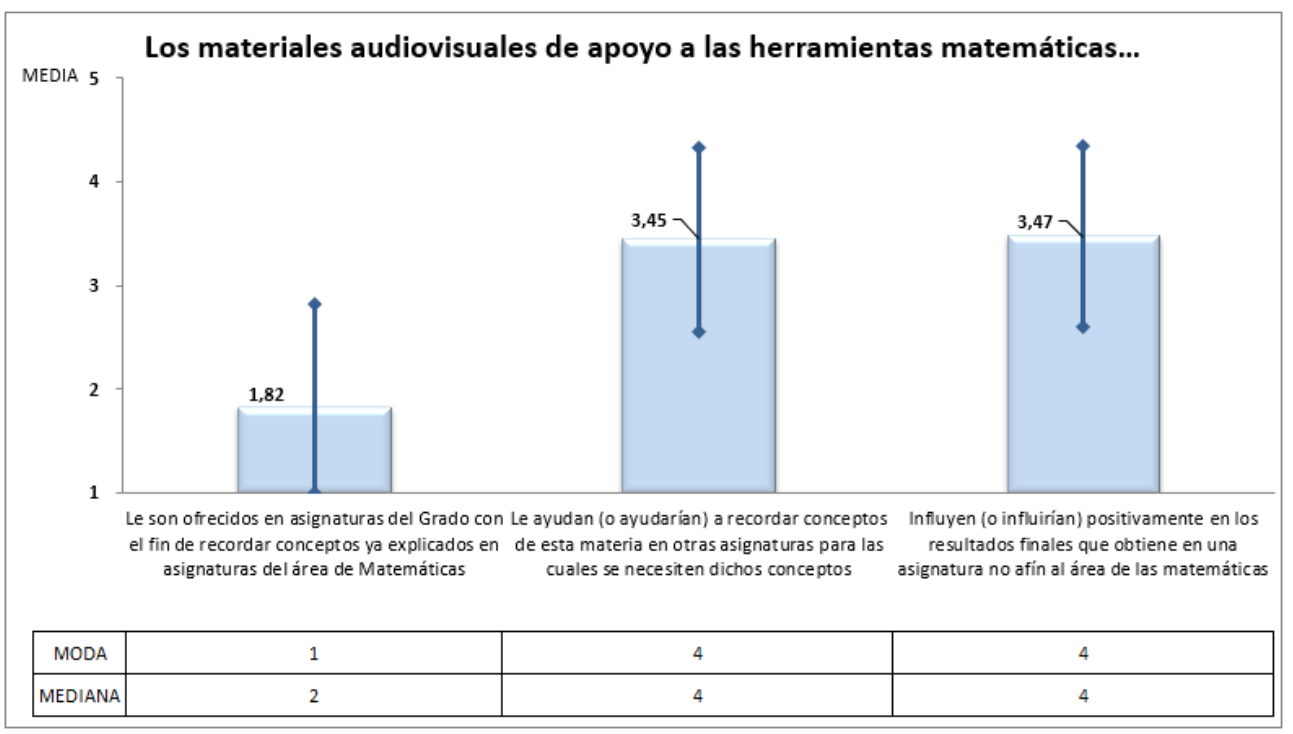

Fig. 4 Resultados de los materiales audiovisuales

Por su parte, se ve una relación muy próxima entre las respuestas a las preguntas de "Le ayudan (o ayudarían) a recordar conceptos de esta materia en otras asignaturas para las cuales se necesiten dichos conceptos" y la pregunta de "Influyen (o influirían) positivamente en los resultados finales que obtiene en una asignatura no afín al área de las matemáticas", lo que indica la relación que ve el alumnado entre asimilar los conceptos y ayudarles a superar positivamente la asignatura.

Para poder valorar el método preferente por el alumnado, se decide representar la Figura 5 y la Figura 6, con la comparativa a las cuatro propuestas, donde se representa la media de 
las respuestas registradas, y la desviación en las respuestas. Respecto a la moda y la mediana, como se representa en las figuras 1 a 4 , se obtiene un valor de 4 en todas estas cuestiones para ambos parámetros, lo que es significativo y muestra la constancia en las respuestas por parte del alumnado donde ven que estas técnicas favorecerían positivamente su aprendizaje.

Las siguientes figuras ilustran la comparativa de las cuatro técnicas en términos de ayudar al estudiante a recordar y emplear los conceptos matemáticos y en términos de influencia positiva en los resultados obtenidos.

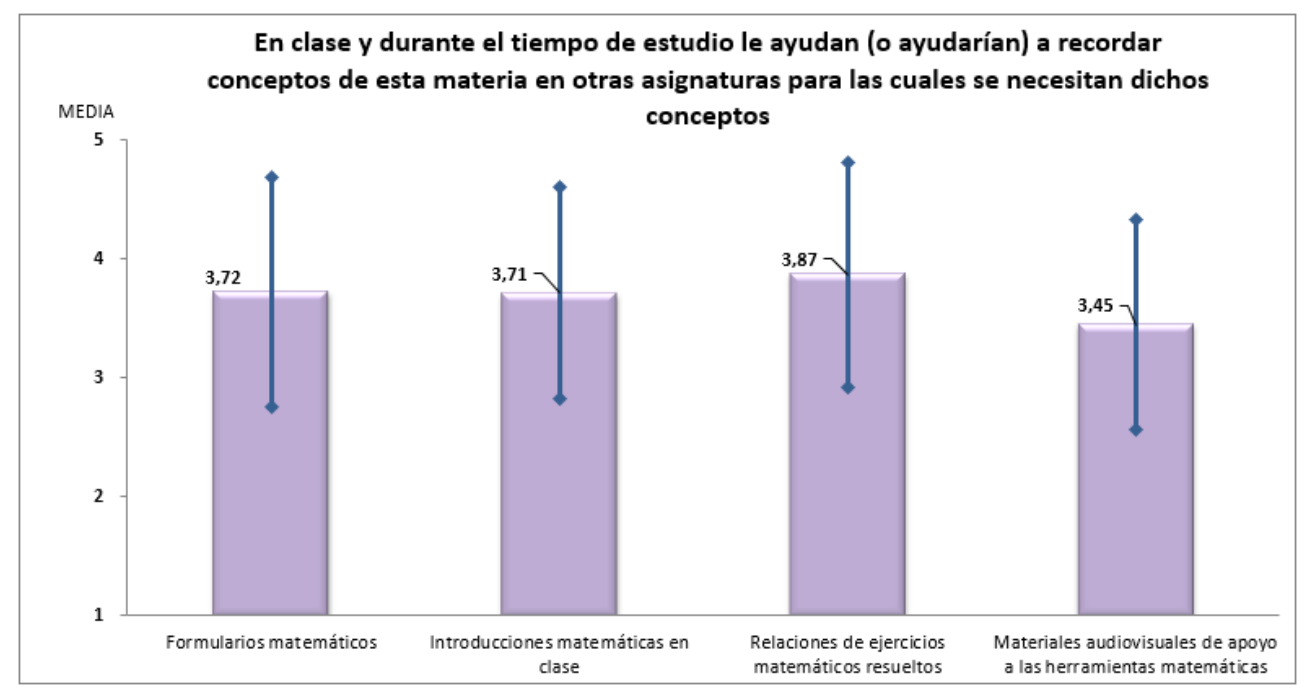

Fig. 5 Valoraciones medias sobre la asimilación de conceptos

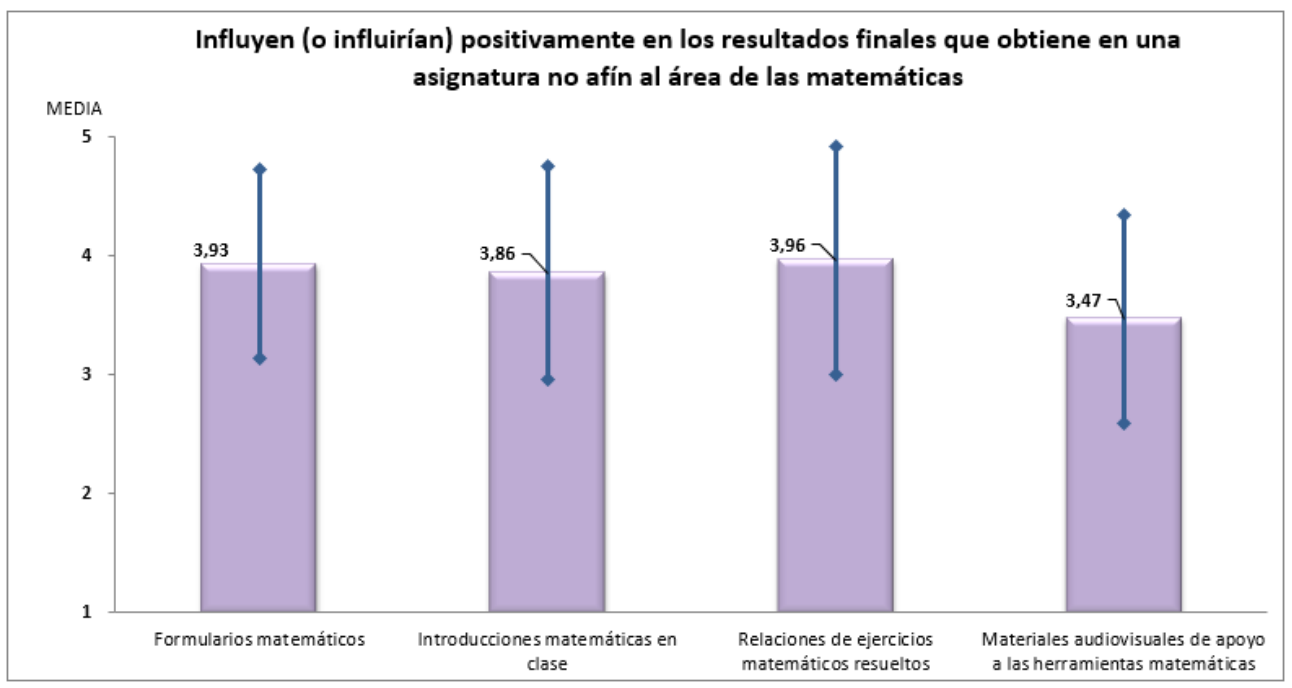

Fig. 6 Valoraciones medias sobre los resultados finales en las asignaturas 
Valorando los resultados expuestos, se aprecia que los estudiantes ven como positivos todos los métodos expuestos, pues todos obtienen una puntuación superior al 3. Si bien, lo medios audiovisuales son los peor valorados, esto se acompaña de comentarios de los alumnos sobre que los videos pueden resultar algo difíciles de seguir, y que están más acostumbrados a los otros métodos para mejorar en sus estudios.

Aunque las relaciones de ejercicios resueltos son el método mejor puntuado, no hay mucha diferencia con respecto a los formularios matemáticos o las introducciones en clase, con lo que se puede desprender que la decisión de tomar una de estar metodologías para mejorar la asimilación de los conceptos matemáticos puede trasladarse al profesor, y su criterio para escoger la que más adecuada sea para la asignatura a impartir, dependiendo de factores como el temario, el alumnado y las preferencias del propio profesor.

\section{Conclusiones}

En la presente investigación se ha introducido la problemática detectada en alumnos de estudios universitarios de la rama técnica, como es el caso de la ingeniería naval y oceánica, donde tiene dificultadas a la hora de la aplicación de sus conocimientos matemáticos en asignaturas que no pertenecen al área propia de las matemáticas. Esto ocasiona que los estudiantes no consoliden las competencias definidas en la titulación, y por lo tanto no obtengan los conocimientos esperados.

Con el objetivo de poder paliar esta circunstancia, este estudio ofrece una serie de 4 técnicas docentes de apoyo al profesor, para transmitir a los estudiantes las necesidades en materia de herramientas matemáticas que pueden ser demandadas en las diferentes asignaturas que componen los planes de estudio de las carreras de ingeniería. Para ello se han planteado las siguientes soluciones didácticas:

- Formularios matemáticos.

- Introducción matemática al principio de las clases.

- Ejercicios matemáticos propuestos y resueltos.

- Material audiovisual.

Se ha realizado una serie de encuestas al alumnado, para así poder valorar cuantitativamente cuál de estas iniciativas ven más adecuada para ellos, obteniendo una valoración positiva en todas ellas, tanto para afianzar los conocimientos, como para poder aprobar con éxito las asignaturas.

Los materiales audiovisuales, que pueden ser en forma de vídeos grabados por el profesor o de contenido digital de alta calidad, ha sido la alternativa peor valorada por los estudiantes, mientras que los ejercicios matemáticos propuestos y resueltos, y los formularios matemáticos se identifican como las opciones preferentes por el alumnado. Por su parte, las introducciones matemáticas al principio de las clases han recibido una buena puntuación, pero hay que indicar, que en la fase de conversaciones no estructuradas con otros docentes y con los propios alumnos se identifica el riesgo de que estas introducciones mermen demasiado tiempo al resto del contenido de la asignatura. 
Con todo ello, se puede concluir que cualquiera de las técnicas, que se enuncian en esta investigación, es recomendable para mejorar la comprensión de los estudiantes, dotándoles de una mayor agilidad a la hora de aplicar los conocimientos matemáticos en el resto de las asignaturas que componen los planes de estudio. El docente podrá elegir entre este abanico de posibilidades según sus propios criterios, pues sin duda debe ser cada profesor el que valore en cada caso cuál de las 4 técnicas se adapta mejor al contenido de su asignatura, al perfil de los estudiantes y a sus preferencias como docente.

\section{Referencias}

ARRAIZ, A. M., SABIRÓN, F., BERBEGAL, A. y FALCÓN, C. (2016). "La evaluación de competencias: el portafolio digital" en La Cuestión Universitaria, vol., issue 8, pp. 140-151.

AVILÉS, R. R., RODRÍGUEZ, G. B. y SOLANO, M. I. M. (2018). "¿ Cómo cambiar las creencias y el pensamiento utilizando los contenidos de la modelación matemática en la formación de ingenieros?(Original)" en Roca. Revista científico-educacional de la provincia Granma, vol. 14, issue 5, pp. 106-117.

BARRERA, M. E. A., PULIDO, H. G. y GONZÁLEZ, P. G. (2018). "Diseño y aplicación de un cuestionario sobre la práctica docente del profesorado de matemáticas en ingeniería y ciencias" en Góndola, Enseñanza y Aprendizaje de las Ciencias: Góndola, Ens Aprend Cienc, vol. 13, issue 1, pp. 33-54.

BRITO-VALLINA, M. L., ALEMÁN-ROMERO, I., FRAGA-GUERRA, E., PARA-GARCÍA, J. L. y ARIAS-DE TAPIA, R. I. (2011). "Papel de la modelación matemática en la formación de los ingenieros" en Ingeniería Mecánica, vol. 14, issue 2, pp. 129-139.

ECHAURI, A. M. F., MINAMI, H. y SANDOVAL, M. J. I. (2014). "La Escala de Likert en la evaluación docente: acercamiento a sus características y principios metodológicos" en Perspectivas docentes, vol., issue 50 .

ETSINO (2019). Memoria para la solicitud de verificación del título de máster universitario de ingeniería naval y oceánica por la Universidad Politécnica de Cartagena, Cartagena (Spain): ETSINO (Escuela Técnica Superior de Ingeniería Naval y Oceánica).

GODINO, J. D., RIVAS, H., ARTEAGA, P., LASA, A. y WILHELMI, M. R. (2014). "Ingeniería didáctica basada en el enfoque ontológico-semiótico del conocimiento y la instrucción matemáticos" en Recherches en Didactique des Mathématiques, vol. 34, issue 2/3, pp. 167-200.

HARRIS, D., BLACK, L., HERNANDEZ-MARTINEZ, P., PEPIN, B., WILLIAMS, J. y TEAM, W. T. T. (2015). "Mathematics and its value for engineering students: what are the implications for teaching?" en International Journal of Mathematical Education in Science and Technology, vol. 46, issue 3, pp. 321-336.

LUACES, V. M. (1998). "Matemática como asignatura de servicio: algunas conclusiones basadas en una evaluación docente" en NÚMEROS. Revista de didáctica de las matemáticas, vol. 36, issue, pp. $65-74$.

MANZUETA CONCEPCIÓN, J. A., MACHADO RAMÍREZ, E. F. y BLANCO SÁNCHEZ, R. (2018). "Debates y perspectivas del proceso de formación y desarrollo de las competencias matemáticas en las carreras de Ingeniería" en Didasc@ lia: Didáctica y Educación, vol., issue 4, pp. $261-274$ 
MASCARAQUE-RAMÍREZ, C. y PARA-GONZÁLEZ, L. (2016). "Propuestas de mejora de la asimilación de los conceptos matemáticos en asignaturas no afines al área por medio de material de apoyo" en: Bolarín Martínez, M. J., Porto Currás, M. y García Hernández, L. Evaluación e identidad del alumnado en Educación Superior. Murcia: Universidad de Murcia.

MENDIBLE, A. y ORTIZ, J. (2007). "Modelización matemática en la formación de ingenieros. La importancia del contexto" en Enseñanza de la Matemática, vol. 12, issue 16, pp. 133-150.

NAPITUPUlU, D., RAHIM, R., ABDUllah, D., SETIAWAN, M. I., ABDILlAH, L. A., AHMAR, A. S., SIMARMATA, J., HIDAYAT, R., NURDIYANTO, H. y PRANOLO, A. Analysis of Student Satisfaction Toward Quality of Service Facility. Journal of Physics: Conference Series, 2018. IOP Publishing, 012019.

POHJOLAINEN, S., MYLLYKOSKI, T., MERCAT, C. y SOSNOVSKY, S. (2018). Modern Mathematics Education for Engineering Curricula in Europe: Springer.

SERNA, E. y FLÓREZ, G. (2013). "El razonamiento lógico como requisito funcional en ingeniería" en Innovation in Engineering, Technology and Education for Competitiveness and Prosperity, vol., issue, pp. 14-16. 